\title{
Technogenic resources for nanotechnologies in construction
}

\author{
Alexander Guryanov ${ }^{1,}$, , Sofia Korenkova $^{1}$, and Lyubov Bezgina ${ }^{1}$ \\ ${ }^{1}$ Samara State Technical University, Institute of Architecture and Civil Engineering, 194, \\ Molodogvardeyskaya St., 443001, Samara, Russia
}

\begin{abstract}
A large group of industrial wastes from water purification, treatment and softening has been considered. The reference is made to the fact that slurries are a unique man-made product technologically ready for use and constantly restocking. Slurries have been classified by conditions of the formation, composition, chemical and physical properties. The nanoscale structural parameters of the slurry particles (the size distribution and fractal dimension) have been determined by the neutron small-angle scattering method. The conducted researches allow slurries to be considered a nanotechnogenic raw material. The use of slurries as additives in the building material production process reduces the harmful influence of industrial wastes on the environment and creates a significant reserve of material and energy resources in the construction industry.
\end{abstract}

\section{Introduction}

Recycling of the industrial waste is an important environmental and economic problem [1]. Among a variety of industrial wastes a special mention should go to a large group of them, i.e. slurries resulting from the chemical treatment of the industrial waste water in machinebuilding, tool engineering, metal working, ferrous and non-ferrous metallurgy, oil production and refining [2]. The formation of the slurry occurs as a result of processes of chemical reactions, dispersion and adsorption, as well as mixing, setting and coagulation of solid particles from supersaturated solutions of the waste water at its cleaning or softening. Conditions for the formation of slurries are sol-gel-assisted. The slurry is a suspension which is characterized by the stable structure and composition.

Most slurry wastes find no practical application. To store them in the dumps is one of the sources of environmental pollution and disruption of the ecological balance [3]. The consequences of the slurry waste negative impact on nature and people can be eliminated by the creation of the developed system of the slurry recycling as one of the components in the production of materials for various purposes [4]. Slurries are a unique man-made product. The use of them as additives increases the strength, moisture resistance and durability of building materials with cement $[2,5]$.

This paper summarizes the results of the classification of the slurry waste. The neutron small-angle scattering method (SANS) [6] was used to determine structural parameters of

* Corresponding author: gurjanovam@mail.ru 
basic types of nanoscale slurries. This definitely makes it possible to consider slurry wastes to be nanotechnogenic raw materials. The qualitative improvement of properties of building materials with cement thanks to slurry additives is due to the fact that they affect the formation of structural parameters of the final product at the micro and nanoscale $[7,9]$.

\section{Materials and methods}

The slurry wastes can be divided into groups according to the following main factors: the condition of the formation, the chemical and mineralogical composition, the action tendency when used as additives to building materials with cement.

The industrial discharged waters are very diverse. At the enterprises of mechanical engineering and energy the waste water formation is associated with washing operations, application of technological mixtures, emulsions and electrolytes. The slurry is the end product of the waste water which has gone through crystallization, settling and water removal. The slurries differ mainly in their chemical and mineralogical composition. We may distinguish mineral and organic- mineral slurries.

The basis of mineral slurries is oxides, carbonates and hydroxides of aluminum, magnesium, iron and silicon. They represent a heterogeneous system in which water is the dispersion medium and solid particles are the dispersed phase.

A distinctive feature of the organic-mineral sludge (oil slurries) is the presence of the residual oil, petrochemical products and emulsified particles of mineral oils in their composition. The composition of some mineral slurries is given in Table 1.

Table 1. Composition of mineral slurries.

\begin{tabular}{|c|c|c|c|c|c|c|c|}
\hline Slurry sample & $\begin{array}{c}\mathbf{A l}_{\mathbf{2}} \mathbf{O}_{\mathbf{3}} \\
{[\mathbf{\%}]}\end{array}$ & $\begin{array}{c}\mathbf{C a O} \\
{[\%]}\end{array}$ & $\begin{array}{c}\mathbf{M g O} \\
{[\%]}\end{array}$ & $\begin{array}{c}\mathbf{F e}_{2} \mathbf{O}_{\mathbf{3}} \\
{[\mathbf{\%}]}\end{array}$ & $\begin{array}{c}\mathbf{S i O}_{2} \\
{[\%]}\end{array}$ & $\begin{array}{c}\mathbf{S O}_{3} \\
{[\%]}\end{array}$ & $\begin{array}{c}\text { Losses during } \\
\text { steaming [\%] }\end{array}$ \\
\hline Carbonate & $0-14$ & $30-48$ & $2-12$ & $2-12$ & $2-5$ & $0-7$ & $32-42$ \\
\hline Aluminum- alkaline & $42-58$ & $0-10$ & $0-5$ & $0-6$ & $0-2$ & $0-7$ & $30-58$ \\
\hline Aluminum-calcium & $12-36$ & $10-21$ & $2-7$ & $1-7$ & $2-8$ & $1-6$ & $34-52$ \\
\hline
\end{tabular}

Despite the variation in the percentage of the individual components of the slurry, the high technological readiness for use and the slurry constant restocking enable to speak about their efficiency in the production of building materials.

In regards to the action tendency slurries can be attributed to filling (e.g. carbonate slurries) or structure-forming additives (e.g. aluminum-alkaline and aluminum-calcium slurries) [7-9].

The hydration of the cement clinker is associated with the formation of the skeleton of the hydrated calcium silicate particles $[10,11]$. The parameters characterizing the structure of the hydrated calcium silicate particles, the ratio of the free and bound water the presence of pores and their shape and size distribution, i.e. the structural parameters of the material at micro and nanoscale [7-9], affect the properties of the resulting cement brick [12, 13]. It is possible to obtain certain specified properties of building materials with cement by using sludge as additives to be fillers or modifiers. It shows up most vividly if there is a nanodispersed component in additives introduced into the cement compositions.

To determine the material structural parameters within the limits from $1 \mathrm{~nm}$ to $100 \mathrm{~nm}$, it is possible to use the neutron small-angle scattering method (SANS) [6]. This method is appropriate to investigate nanoparticles of different physical nature. It is also successfully used to study at the nanoscale a variety of nanodispersed materials and to determine structural parameters of nanomaterials, as well as bulk ones containing nanodispersed components, e.g. hydrated cement compositions [14-16]. 
The SANS intensity $I(q)$ depends on the scattering power of the compositional inhomogeneity of the matter and the contrast at the phase boundary. It is the function of the neutron pulse sent at scattering

$$
q=\frac{4 \pi}{\lambda} \sin \frac{\theta}{2}
$$

where $\theta$ is the angle of neutron scattering.

The SANS intensity $I(q)$ carries information about the structure of scattering particles (scattering centres) over the atom. Provided that $q R_{c}<1$ (Guinier regime), the intensity of the small-angle scattering $I(q)$ is determined by the average (characteristic) size and shape of scattering particles:

$$
I(q)=I(0) \exp \left(-\frac{R_{g}^{2} q^{2}}{3}\right),
$$

where $R_{g}$ is the particle radius of gyration. From the experimental data on small-angle scattering in the Gunier regime it is possible to assess the particle radius of gyration $R_{g}$ and their typical size $R_{c}$.

If the condition $q R_{c}>1$ (Porod regime) for the intensity of the small-angle scattering $I(q)$ on fractal objects has been fulfilled, there happens to be a power characteristic from the transmitted neutron pulse:

$$
I(q) \propto q^{-n} .
$$

The deviation of the exponent $n$ from the Porod asymptotic behavior $(n=4)$ indicates fractal properties of scattering objects and makes it possible to determine their fractal dimension. In the case of volumetric or mass fractals the exponent coincides with the fractal dimension $n=D_{V}$ and takes on the value from the interval $1<D_{V}<3$. In the case of scattering by objects with a fractal surface, the fractal dimension is $D_{S}=6-n$ it takes values from the interval $2<D_{S}<3$. The exponent is respectively in the interval $3<n<4$.

Thus, by the slope of log-log linear segments of the SANS dependencies it is possible to determine the scattering objects of a particular fractal type (volume or surface) and the fractal dimension [17-19].

The wavelength $\lambda$ of slow neutrons in the neutron small-angle scattering method is in the interval which allows determining structural parameters of scattering particles in the nanometer interval and to clearly relate test materials to nanomaterials.

For polydisperse systems according to small-angle scattering we can recover the distribution function of the scattering objects in terms of sizes $G(R)$ on the basis of the spectra of pair correlations $\chi(R)$ obtained by the Fourier transformation of the data for cross sections of neutron scattering $\sigma(R)$. This function is related to the shape of the particles and describes quantitatively the set of segments connecting elements of the particle volume and depends on the distribution of inhomogeneities inside the particles. The size distribution function $G(R)$ has the following form:

$$
G(R)=R^{2} \gamma(R)=\frac{1}{2 \pi^{2}} R^{2} \int \frac{d \sigma(q)}{d \Omega} \frac{\sin (q R)}{q R} q^{2} d q .
$$

The function $G(R)$ shows if there are particles of the given size (radius) in the test material. All SANS experimental data is implemented using the software ATSAS 2.8.0 [20, 21]. 


\section{Results}

The SANS intensity spectra from the sludge samples were measured using the diffractometer "Membrana-2" installed at the reactor WWR-M in the Petersburg Nuclear Physics Institute (Gatchina) [22]. The wavelength of slow neutrons was equal to $\lambda=0,3$ $\mathrm{nm}$. The range of the recorded transmitted neutron pulses $q$ varied from $0,03 \mathrm{~nm}^{-1}$ to 0,8 $\mathrm{nm}^{-1}$. The samples of scattered neutrons were recorded in the range of angles $\theta= \pm 0,017$ rad. The detector consisted of forty-one ${ }^{3} \mathrm{He}$-counters. The intensity of the beam on the sample was measured by two counters (monitors) installed in front of the sample above and below the axis of the neutron beam.

The samples of the test sludge were placed in the target device that is a cavity in a cadmium plate. The volume of the cavity equaled $0,52 \mathrm{sm}^{3}$. The sample layer thickness was equal to the thickness of the cadmium plate $d_{\mathrm{S}}=2 \mathrm{~mm}$. Loose materials were placed in the cavity of the target device and covered with an aluminum foil of $6 \mathrm{mkm}$. The transmission coefficient of the neutron beam was $T=0,45-0,85$.

The SANS data resulting from the experiment were normalized in accordance with the monitor readings and the transmission intensity. We also conducted the subtraction of background and contribution of neutrons that had passed through the sample without scattering. The obtained distributions of scattering intensities $I_{\mathrm{S}}(q)$ were normalized to those obtained under the similar conditions of the intensity distribution $I_{\mathrm{ST}}(q)$ of the standard sample which was the light water layer $d_{\mathrm{ST}}=1 \mathrm{~mm}$. Differential cross sections of the sample scattering based on the $1 \mathrm{sm}^{3}$ of the sample volume in absolute units were calculated according to the following formula

$$
\frac{d \sigma(q)}{d \Omega}=\frac{I_{S}(q)}{I_{S T}(q)} \frac{d_{S T}}{d_{S}} \frac{d \sigma_{S T}}{d \Omega} .
$$

From differential cross sections of neutron scattering the distribution function of the scattering center (particles) sizes $G(R)$ in the approximation of homogeneous spheres was recovered using the Fourier transform.

Analyzing the experimental data we selected the maximum radius of the scattering objects. When its value was $50 \mathrm{~nm}$, the points corresponding to minimum neutron pulses transferred during the experiment collapsed (were not described theoretically).The points corresponding to maximum pulses transferred collapsed when the radius value was $100 \mathrm{~nm}$. The description of the SANS experimental data was correct selecting the maximum size of scattering objects equal to about $80 \mathrm{~nm}$. The average radius of scattering objects for test samples was in the range from $30 \mathrm{~nm}$ to $65 \mathrm{~nm}$. 


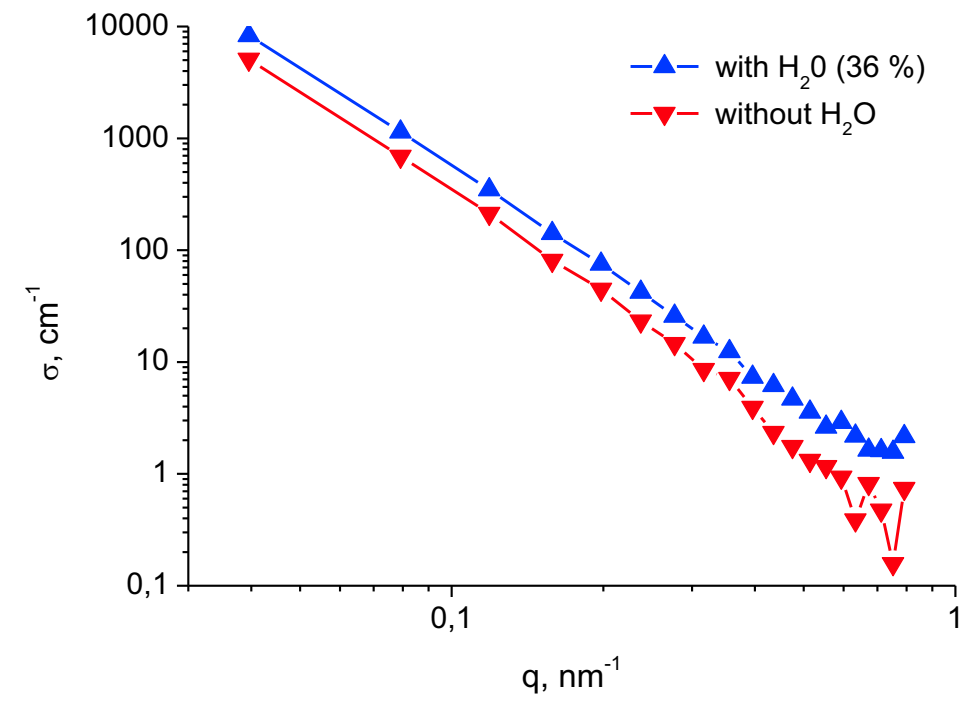

Fig. 1. Experimental spectra of the SANS differential cross sections for the carbonate sludge in two states (wet and dry).

Figure 1 shows spectral plots of the differential cross sections of the neutron scattering of the carbonate sludge sample. Figure 2 shows graphs of the calculated distribution functions of the scattering objects by sizes for the carbonate sludge sample.

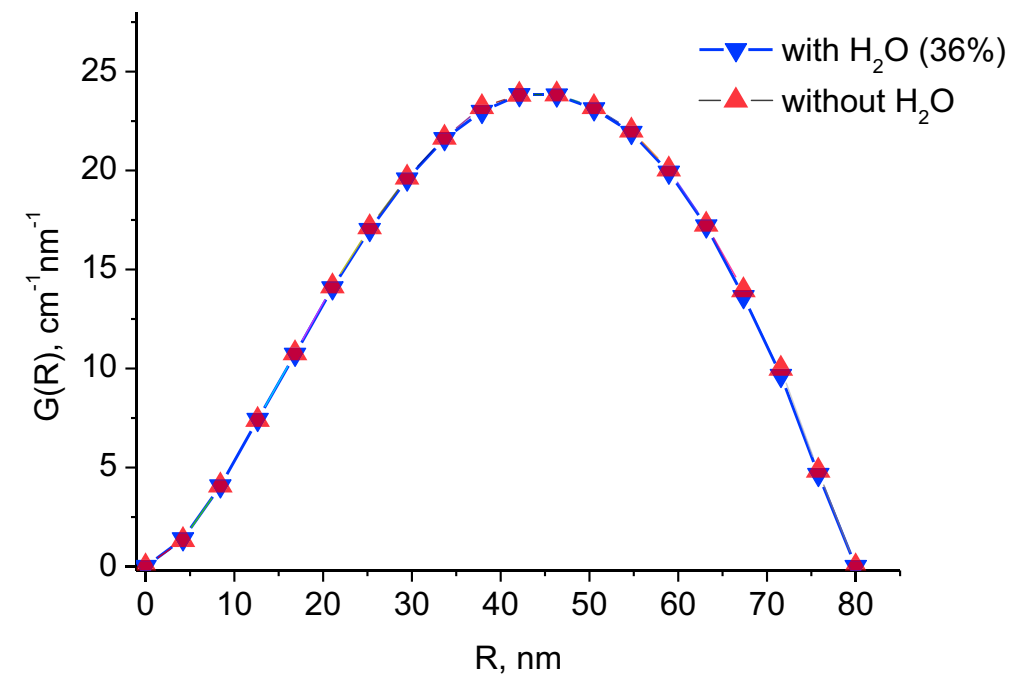

Fig. 2 The distribution function of scattering objects by sizes for the carbonate sludge sample in two states (wet and dry).

According to experimental data of neutron scattering intensities the following calculations were performed: the average radii were found using formula (2); formula (3) was used for fractal dimensions; formula (4) for differential scattering cross sections and formula (5) for scattering object distribution functions by sizes.

For all sludge samples it was possible to observe the power dependence of the neutron scattering intensity from the transmitted neutron pulse. Table 2 shows the description of test sludge samples, the average size of particles $R_{c}$, and values of the fractal dimension $D_{S}$. 
Table 2. Structural parameters of sludge samples.

\begin{tabular}{|c|c|c|c|}
\hline $\begin{array}{c}\text { Sample } \\
\text { number }\end{array}$ & Slurry type & $\boldsymbol{R}_{\boldsymbol{c}}, \mathbf{n m}$ & $\boldsymbol{D}_{\boldsymbol{S}}$ \\
\hline 1 & Carbonate (wet, $\left.36 \% \mathrm{H}_{2} \mathrm{O}\right)$ & 45 & 2,61 \\
\hline 2 & Carbonate(dry) & 47 & 2,65 \\
\hline 3 & Aluminum-alkaline(wet, $\left.38 \% \mathrm{H}_{2} \mathrm{O}\right)$ & 38 & 2,54 \\
\hline 4 & Aluminum- alkaline(dry) & 40 & 2,58 \\
\hline 5 & Aluminum-calcium(wet, $\left.35 \% \mathrm{H}_{2} \mathrm{O}\right)$ & 44 & 2,56 \\
\hline 6 & Aluminum-calcium(dry) & 46 & 2,60 \\
\hline
\end{tabular}

\section{Discussion}

The SANS method was used to determine at the nanoscale structural parameters of some basic types of slurries. The obtained results show that slurries are composed of the nanodispersed component and it is quite possible to refer them to nanotechnogenic raw materials. A slight change in the structural parameters of slurries at various stages of completion (wet or dry) demonstrates their stability.

\section{Conclusions}

Slurry waste resulting from the industrial enterprise sewage treatment are nanotechnogenic raw materials. The slurries affect the formation of structural parameters of building materials with cement at the micro and nanoscale acting as fillers or modifying additives, efficiently and purposefully improving their properties

Furthermore, the use of the slurry waste for the production of building materials makes it possible to solve the important environmental and economic problem of the industrial waste utilization, to avoid environmental pollution and disruption of the ecological balance.

\section{References}

1. K.L. Chertes, A.A. Savelyev, E.G. Martynenko, O.V. Tupitsyna, A.A. Mikhasek, Urban Constrution and Architecture 1, 49-57 (2016) DOI: 10.17673/Vestnik.2016.01.8

2. S.F. Korenkova, T.V. Sheina, The basics and the concept of chemical precipitation of the industrial waste in the construction industry (SGASU, Samara, 2004)

3. K. Chertes, O. Tupitsyna, V. Pystin, A. Savelyev, E. Martynenko, MATEC Web of Conferences 86, 06003 (2016)

4. K.L. Chertes, O.V. Tupitsyna, V.N. Pystin, A.M. Shterenberg, Urban Construction and Architecture 4, 78-84 (2015) DOI: 10.17673/Vestnik.2015.04.10

5. A.M. Guryanov, Industrial and civil engineering 6, 55-58 (2015)

6. M.V. Avdeev, V.L. Aksenov, Advances in physical sciences 180-10, 1009-1034 (2010)

7. A.M. Guryanov, Procedia Engineering 111, 283-289 (2015)

8. A.M. Guryanov, Procedia Engineering 153, 217-222 (2016)

9. A. Guryanov, S. Korenkova, Yu. Sidorenko, MATEC Web of Conference 86, 04011 (2016) DOI: 10.1051/matecconf/20168604011

10. J.W. Bullard, H.M. Jennings, R.A. Livingston, A. Nonat, G.W. Scherer, J.S. Schweitzer, K.L. Scrivener, J.J. Thomas, Cement and Concrete Research 41, 1208$1223(2011)$

11. A. Nonat, Cement and Concrete Research 34, 1521-1528 (2004) 
12. L.B. Skinner, S.R.Chae, C.J. Benmore, H.R. Wenk, P.J.M. Monteiro, Physical Review Letters 104, 195502 (2010)

13. L. Raki, J. Beaudoin, R. Alizadeh, J. Makar, T. Sato, Materials 3, 918-942 (2010)

14. J.J. Thomas, J.J. Chen, A.J. Allen, H.M. Jennings, Cement and Concrete Research 34, 2297-2307 (2004)

15. A.J. Allen, J.J. Thomas, H.M. Jennings, Nature Materials 6, 311-316 (2007)

16. A.J. Allen, J.J. Thomas, Cement and Concrete Research 37, 319-324 (2007)

17. T. Ficker, Theoretical and Applied Fracture Mechanics 50,167-171 (2008)

18. Q. Zeng, M. Luo, X. Pang, L. Li, K. Li. Applied Surface Science 282, 302-307 (2013)

19. Y. Gao, J.Jiang, G.D. Schutter, G. Ye, W. Sun, Construction and Building Materials 69, 253-261 (2014)

20. P.V. Konarev, M.V. Petoukhov, V.V. Volkov, D.I. Svergun, J. Appl. Cryst. 39, 277286 (2006)

21. M.V. Petoukhov, D. Franke, A.V. Shkumatov, G. Tria, A.G. Kikhney, M. Gajda, C. Gorba, H.D.T. Mertens, P.V. Konarev and D.I. Svergun, J. Appl. Cryst. 45, 342-350 (2012)

22. V.M. Lebedev, B.T. Lebedev, I.N. Ivanova, M.R. Kolhidashvili, D.N. Orlova, The analytical measuring system for the small-angle diffractometer "Membrane-2", Preprint PNPI-2785 (2008) 